\title{
Forged Multinational Currency Identification and Detection System using Deep Learning Algorithm
}

\author{
Megha Jadhav \\ Dept. of Computer Sci. \& Engg. \\ Shri JJT University \\ Chudela , Jhunjhunu, Rajstan
}

\author{
Yogeshkumar Sharma, PhD \\ Dept. of Computer Sci. \& Engg. \\ Shri JJT University \\ Chudela, Jhunjhunu, Rajstan
}

\author{
G. M. Bhandari, PhD \\ Dept. of Computer Sci. \& Engg. \\ Shri JJT University \\ Chudela , Jhunjhunu, Rajstan
}

\begin{abstract}
Now a days as many counterfeit banknotes are manufactured and circulated in global market, which results in significant damage and harm to society. Recognizing the currency's originality is a very difficult task to general person. Because of advancement in generating highly accurate fake currency. There are several techniques available, such as automatic sorting of banknotes in payment facilities, automated payment machines or sales machines, which consists of several tasks such as identification of banknote type, classification of recirculation fitness and detection of fake banknotes. Banknote identification is the most important approach, based on an image processing system. There are many techniques used in the classification of banknotes by different countries that has been conducted experiments on separate image data sets of each country. Deep learning is a machine learning technique which analyzes $\&$ learns the features of the original note. Using the neural networks, the most important aspect is to find more essential features. In the age of big data, in which vast amounts of data must be processed for any application in the real world, the superior techniques are deep learning. In this study, banknotes from various countries are examined by extracting their minute features in carefully and analyzing them using deep learning. Proposed system recommended a Convolutional Neural Network algorithm to detect Forged banknote using dataset of multiple country currency. This approach is chosen to achieve high accuracy with good performance with respect to loss and accuracy in training and validation in terms of huge dataset. So it helps individuals to avoid personal economic damage caused by counterfeiters.
\end{abstract}

\section{Keywords}

Deep Learning, Currency Recognition, Currency Identification

\section{INTRODUCTION}

Now everybody works online because of their busy lives, no one is bothered to visit a bank as majority of financial transaction are performed using various ways introduced on internet. The rise in electronic and digital financial transactions resulting in a decline use of physical currency, still transactions involving banknotes is the universal means of exchange or general circulation in order to facilitate the sale and distribution of goods in both everyday life and largescale commerce. Currency within the system of market economy Automated machines include many techniques in these transactions and have the ability to handle multiple tasks, including the identification of banknotes, fitness verification, counterfeit detection and serial number recognition. Problems arise when banknotes used in systems like automated teller machines (ATMs) and vending machines cannot be recognized because of higher than average spoilage levels.
Currency reputation is critical as a means of economic activity as it destroys not only personal property but also national creditworthiness. Cases that have recently been falsified are increasing rapidly in forgery cases. Counterfeiting crime is not just one country's concern. Hence detecting counterfeit is important for economic and social soundness.

The development of automated systems for recognizing currencies has developed day by day. In many areas such as the banking system, railway ticket counter, shopping mall, currency exchange service etc., cost-effective solution and efficient currency recognition system is considered as important. Banknotes are used to carry out finance operations. The various technological advances and new equipment in the field of scanners and printing machines have led the miscreants to make high accurate copies of banknotes. It is very difficult for human eyes to differentiate between fake and genuine because they are designed with great precision to look like a genuine note and counterfeiters are prone to making such fake currencies, and appear to have overwhelmed the network. This scenario enforced there is a requirement of a proper technique that helps to result properly with lot of training data to achieve high accuracy. Proposed system helps to common people to deal with this scenario easily using image of currency. Mainly this paper focused on multiple countries with different denominations of that country. Performance of system can be monitored with the help of precision and time. The primary objective is to focus on minute parameter which helps to classify between genuine or fake banknotes and introduce a straightforward and competent approach.

\section{LITURATURE SURVEY}

A lot study is introduced by various researchers in classification and identification of banknotes of different countries and also conducted various experiment on image dataset of each country.

Achal Kamble et al [1] proposed a new method for the identification of fake Indian documents by making use of image processing techniques. Author working on currency image is the one represented in the space of dissimilarity, that dimensional testing of the disparity between the picture being considered and a prototype. They have obtained the dissimilarity between two images; detecting and defining the local key points on each image.

Tushar Agasti et al [2] introduced a new method for detecting Indian currency notes using image processing. Feature extraction includes features such as serial number, protective string, identification mark, portrait of Mahatma Gandhi. Proposed method also extracts functionality, even if the note has scrawl on it. 
Monali Patil et al [3] is introducing a new approach to detecting fraud on Indian Notes. First, take the image input and pre-process the image after pre-processing, and apply algorithm to extract the image's inner and outer edges. Clustering is achieved using the k-means algorithm, comparing the features of the image and classifying it as original or fake using SVM algorithms. Experimental results show that using SVM Algorithm has better accuracy performance than using the CNN Algorithm for Indian Currency.

Gai et al [4] introduced method which uses quaternion wavelet transform and generalized Gaussian density. These techniques are used to extract the function and Neural Network is used to classify banknote in China, Europe and United State.

A distance based Euclidean banknote system is proposed by Bhurke et al [5] Developed strong GUI for different country including India, Saudi Arabia, Australia, etc.

Based on above literature survey, we analyzed that we should concentrate on precision and time to hand huge data set of multiple country with different denominations of each country.

\section{PROPOSED METHODOLOGY}

General flow of proposed system for banknote recognition is as shown in following figure 1 Following are the steps used to perform analysis.

Step 1: Take Input as Inage of front view and back view of banknote.

Step 2: Perform preprocessing on image to rescale size, Shearing and transformation to zoom.

Step 3: Transfer processed image to pre-trained CNN.

Step 4: Categorize Image into genuine and fake banknote and determine denomination.

Firstly dataset of various countries are generated which includes India, Bhutan, and Saudi Arabia initially. Dataset is generated from various sources of internet, Churan Bank, Children Bank of India etc. These images are collected for both front side view and back side view of banknotes. After dataset generation it is categorized into two parts as genuine and fake for the purpose if training [6].

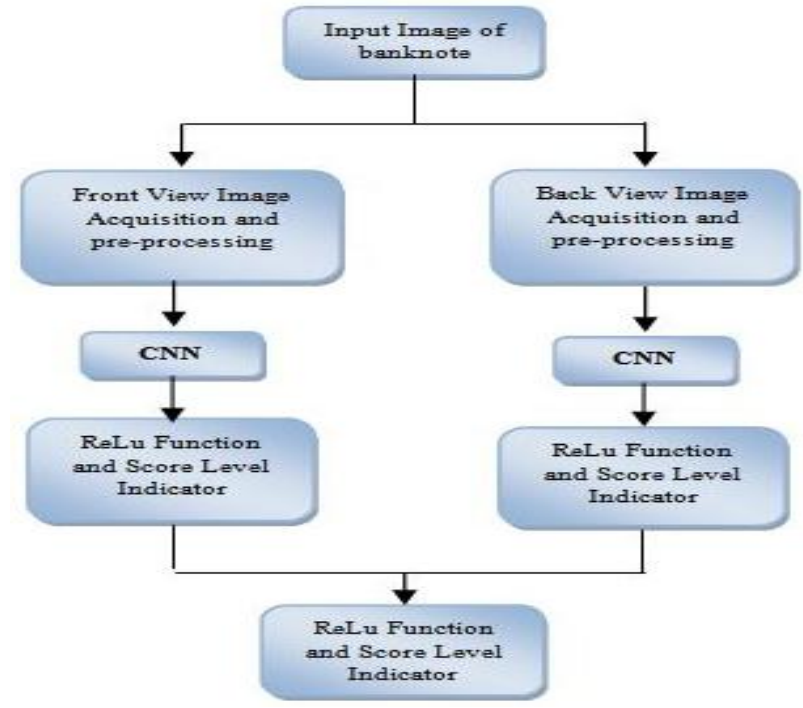

Fig 1: General Flow of Proposed System
Pre-processing of images is implemented to strengthen and intensify certain extracted image characteristics important for future analysis and processing. Using median filter, noise from image is eliminated from the separated image. Median filter is effectively based on a moving window over the entire image and measuring the resulting pixel value as the median value of the present window's brightness value. Restore the resulting smoothed picture canals. Another pre-processing implemented is to normalize the scale of different currency notes with the same aspect ratio retained. The aspect ratio may be defined as the ratio of note width to note height. Complete image size the aspect ratio of the note of a given denomination is independent of the distance from which the photograph was taken [7]-[8].

Alex Net architecture inspired the CNN used in proposed method. Alex Net consists of eight layers. Top five layers are convolutional layer where some of them are accompanied by max-pooling layer. Bottom three layers are fully connected layers. Here ReLu is used as a activation features to get improved training efficiency over tanh and sigmoid [3]. The main advantage of using ReLu is there is a decreased risk of gradient loss. In Convolutinal layer feature extraction is done in several filters with different sizes followed by pooling and in fully connected layer classification is done with number of nodes at each layer. So technically this architecture is used for training and testing purpose where each input image pass through series of layers with different size filters, pooling and fully connected layers series. Filter coefficient of convolution layer and weights of fully connected layers are learned from training data of images of banknotes. In network training phase full network models trained coefficient and weight in memory for the purpose of testing. Finally in proposed method, as an output banknote is classified as either genuine or fake according to origin of country and denomination using fully connected layers of trained convolutional network model [9]-[10].

\section{PRAPOSED IMPLEMENTATION}

In this study the currencies of Indian, American, Saudi Arabia, and Bhutan are studied and established. For front view and back view, the features of those four currencies of different types are extracted. Segmentation of images in the RGB, HSV and $\mathrm{YCbCr}$ Color regions provides the best comparison of hidden features of all currencies. Following Steps are performed which passes through series of layers as shown in Figure 2.

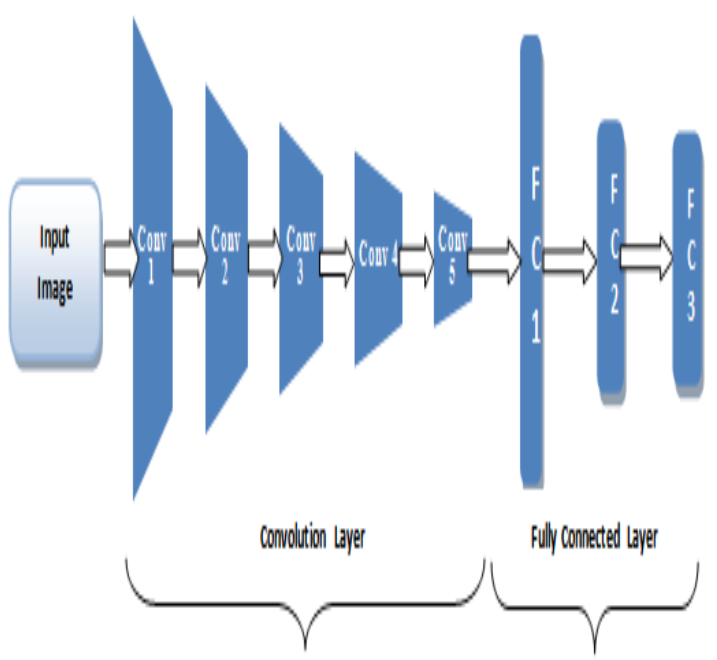

Fig 2: Convolution Neural Network Architecture 
A. Take input image of front side and back side of the currency.

B. In pre-processing phase Images are converted into $\mathrm{RGB}, \mathrm{HSV}$ and $\mathrm{YCbCr}$ formats.

C. Feature extraction on both images is compared with existing data features of currency which have been already stored in the database.

Image is preprocessed by using our deep learning algorithm.

- Segments of currency as an input.

- Set different weights (As Deep learning is work on different layers and it need to set weight for each layer).

- Calculated, Hidden layer by the formula.

$$
\begin{gathered}
\text { input_of_hidden_layerl } \begin{array}{c}
\text { values of original currency } \\
\text { currency }
\end{array} \\
\text { features-inputted } \\
\text { feature }
\end{gathered}
$$

- Using above formula we have calculated 3 layers and using following formula we have calculated error function which helps to take the final decision.

Error fun $=a b s\left(\sum\left(\sum\right.\right.$ output_of_hidden_layer 3$\left.)\right)$....(3) currency and error function comparison decision is taken place

Attributes of paper currencies that are used by citizens to distinguish various banknote denominations are employed in the proposed system. For their identification, the specifics of the picture and the exact characteristics of banknotes, rather they find the common characteristics of banknotes such as the size, background color (the basic color), and the texture present on banknotes. Thus we are introducing some other way or traditional currency detection features [11,12].

\section{RESULTS}

The experiments were performed to identify the Indian, American, Bhutan and Saudi Arabia's fake currency notes. The images and then features were extracted from the acquired images using the proposed technique.

The identification between real image and fake currency image was done on the basis of dissimilarity and discontinuity between them. The extracted features were used for fake currency detection. The decision weather a note is fake or real was made by comparing the values of Original note and error function which have been implemented. Here some images of currencies of different countries has been collected and their features that have been considered for the identification and detection of fake note. Every note is converted into three different types of color image such as RGB, HSV, and YCbCr.

- With the help of values of original features of
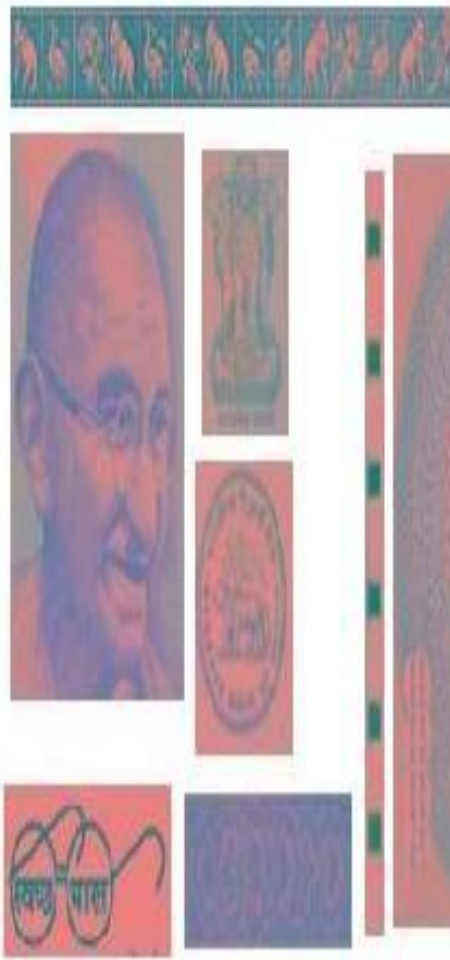

$\mathrm{YCbCr}$
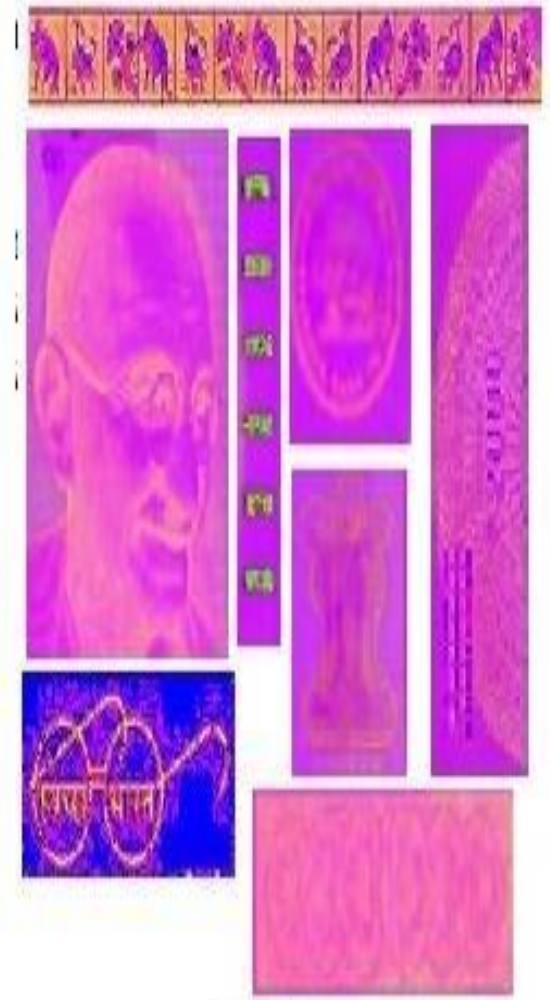

HSV
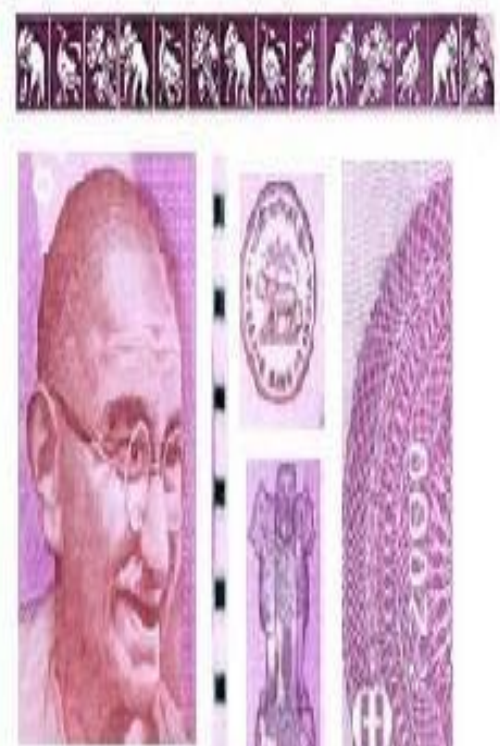

1

(1)

1

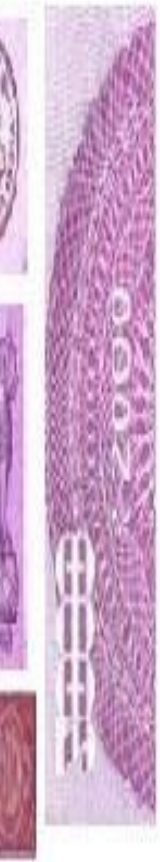

RGB

Fig 3: Different feature Segments Indian Currency 2000

As shown in above Figure 3 eight segments features of each type of color image have been considered for detecting the Indian currency 2000. 


\begin{tabular}{|c|c|c|c|c|c|}
\hline MR1 & MG1 & MB1 & MM1 & SD1 & Corr1 \\
\hline 178.58673 & 104.00663 & 122.88265 & 135.15867 & 39.074955 & 0.9613636 \\
\hline 195.04688 & 182.56275 & 204.10935 & 193.90633 & 80.844159 & 0.9980157 \\
\hline 223.10833 & 192.60095 & 220.51286 & 212.07405 & 40.778547 & 0.9846653 \\
\hline 196.91354 & 150.45472 & 204.04762 & 183.80529 & 43.653317 & 0.9835227 \\
\hline 210.60651 & 145.06523 & 177.7786 & 177.81678 & 45.993545 & 0.9804845 \\
\hline 141.64178 & 104.69193 & 129.81639 & 125.38337 & 77.063507 & 0.9945765 \\
\hline 216.02963 & 212.14014 & 213.91541 & 214.0284 & 80.422073 & 0.9982264 \\
\hline 203.58661 & 158.73637 & 198.65435 & 186.99244 & 41.158766 & 0.9799201 \\
\hline 195.69 & 156.28234 & 183.96465 & 178.64567 & 56.123609 & 0.9850968 \\
\hline MR2 & MG2 & MB2 & MM2 & SD2 & Corr2 \\
\hline 0.9574155 & 0.4245317 & 0.7003401 & 0.69440958 & 0.2263526 & 0.9517122 \\
\hline 0.7293997 & 0.2157469 & 0.8051186 & 0.5834217 & 0.3556505 & 0.9825243 \\
\hline 0.8391649 & 0.1558001 & 0.8848693 & 0.6266114 & 0.3457881 & 0.9768316 \\
\hline 0.8102322 & 0.2724594 & 0.8007331 & 0.6278082 & 0.2687705 & 0.9749145 \\
\hline 0.9078032 & 0.3258784 & 0.8294085 & 0.6876967 & 0.2739342 & 0.9911289 \\
\hline 0.8475181 & 0.385639 & 0.5564682 & 0.5965418 & 0.3094041 & 0.9784295 \\
\hline 0.4184915 & 0.0931764 & 0.8482217 & 0.4532965 & 0.4478486 & 0.9791143 \\
\hline 0.8372695 & 0.238536 & 0.8024577 & 0.6260877 & 0.293375 & 0.9695442 \\
\hline 0.7934118 & 0.263971 & 0.7784521 & 0.611945 & 0.3151405 & 0.9755249 \\
\hline MR3 & MG3 & MB3 & MM3 & SD3 & Corr3 \\
\hline 126.32075 & 125.22415 & 159.41514 & 136.98668 & 19.583366 & 0.8901399 \\
\hline 178.10127 & 135.61969 & 131.94164 & 148.5542 & 43.944808 & 0.9959523 \\
\hline 191.9781 & 135.73619 & 139.41881 & 155.71103 & 32.277513 & 0.9290347 \\
\hline 162.39029 & 144.64482 & 144.57796 & 150.53769 & 20.308719 & 0.941935 \\
\hline 160.61684 & 132.65453 & 154.45179 & 149.24105 & 22.11374 & 0.9700315 \\
\hline 117.85098 & 133.55147 & 142.43053 & 131.27766 & 39.955349 & 0.9621166 \\
\hline 199.32605 & 128.17252 & 129.57921 & 152.35926 & 52.155343 & 0.9876469 \\
\hline 167.74965 & 138.88748 & 144.84197 & 150.49303 & 23.19323 & 0.8748563 \\
\hline 163.04174 & 134.31136 & 143.33213 & 146.89508 & 31.691508 & 0.9439641 \\
\hline & & & & & \\
\hline
\end{tabular}

Fig 4: Data Table of original Indian Note 2000

Here, mean of each values are shown in Figure 4 and 5 MR1,MG1,MB1,MM1 SD1 and Corr1 (Mean Red, Mean

Green, Mean Blue, Mean of Mean RGB, Standard Deviation and Correlation of RGB Image) have calculated for the processing of image on Indian currency 2000 rupees note. Same way calculated this for $\mathrm{YCbCr}$ and HSV image also.

\begin{tabular}{|c|c|c|c|c|c|}
\hline MR1 & MG1 & MB1 & MM1 & SD1 & Corr1 \\
\hline 146.1898 & 57.945918 & 58.35068 & 87.495465 & 66.816878 & 0.0065474 \\
\hline 239.40014 & 221.06629 & 221.17875 & 227.21506 & 23.804585 & -0.0531377 \\
\hline 237.80071 & 222.05381 & 220.74405 & 226.86619 & 8.0656407 & -0.1042158 \\
\hline 206.31447 & 154.7606 & 166.21961 & 175.76489 & 52.293003 & 0.2625157 \\
\hline 192.09562 & 108.46571 & 117.58194 & 139.38109 & 65.870037 & 0.5305986 \\
\hline 157.70207 & 86.37334 & 97.937063 & 114.00416 & 74.812894 & 0.0238581 \\
\hline 214.49295 & 212.89857 & 202.86643 & 210.08598 & 59.662681 & 0.0708941 \\
\hline 200.96557 & 136.14284 & 152.98835 & 163.36559 & 68.098856 & 0.3127183 \\
\hline 199.37017 & 149.96338 & 154.73336 & 168.0223 & 52.428072 & 0.1312223 \\
\hline MR2 & MG2 & MB2 & MM2 & SD2 & Corr2 \\
\hline 0.2857111 & 0.6783843 & 0.5732933 & 0.5124629 & 0.3393087 & 0.09028 \\
\hline 0.4970677 & 0.0799768 & 0.9388241 & 0.5052895 & 0.4538967 & 0.0658168 \\
\hline 0.0172601 & 0.0717777 & 0.9325518 & 0.3405299 & 0.4217212 & 0.0216746 \\
\hline 0.8644332 & 0.2807381 & 0.8091379 & 0.6514364 & 0.3419953 & 0.4577126 \\
\hline 0.7950267 & 0.4782928 & 0.7533167 & 0.6755454 & 0.3028273 & 0.4265379 \\
\hline 0.939082 & 0.540748 & 0.6184395 & 0.6994232 & 0.295653 & 0.0871839 \\
\hline 0.1506254 & 0.0875247 & 0.844696 & 0.3609487 & 0.3772939 & -0.0144631 \\
\hline 0.6690241 & 0.376172 & 0.7881003 & 0.6110988 & 0.3509507 & 0.5199561 \\
\hline 0.5272788 & 0.3242018 & 0.7822949 & 0.5445918 & 0.3604559 & 0.2068374 \\
\hline MR3 & MG3 & MB3 & MM3 & SD3 & Corr3 \\
\hline 88.464796 & 115.01735 & 166.70442 & 123.39552 & 41.967708 & 0.0257869 \\
\hline 210.55694 & 125.19108 & 136.16062 & 157.30288 & 39.486557 & -0.0457038 \\
\hline 210.61548 & 125.44071 & 135.00762 & 157.02127 & 38.116348 & 0.0142072 \\
\hline 163.24594 & 125.41662 & 149.7789 & 146.14715 & 30.054621 & 0.1176694 \\
\hline 131.51909 & 119.61288 & 164.06653 & 138.3995 & 33.675969 & 0.530166 \\
\hline 109.62867 & 122.51493 & 158.49368 & 130.21243 & 40.207356 & 0.0449528 \\
\hline 198.26762 & 123.07885 & 129.32354 & 150.22334 & 45.113074 & 0.0667236 \\
\hline 151.20647 & 125.87364 & 155.22897 & 144.10303 & 35.949774 & 0.2107191 \\
\hline 157.93813 & 122.76826 & 149.34554 & 143.35064 & 38.071426 & 0.1205651 \\
\hline & & & & & \\
\hline
\end{tabular}

Fig 5: Data Table of Fake Indian Note 2000

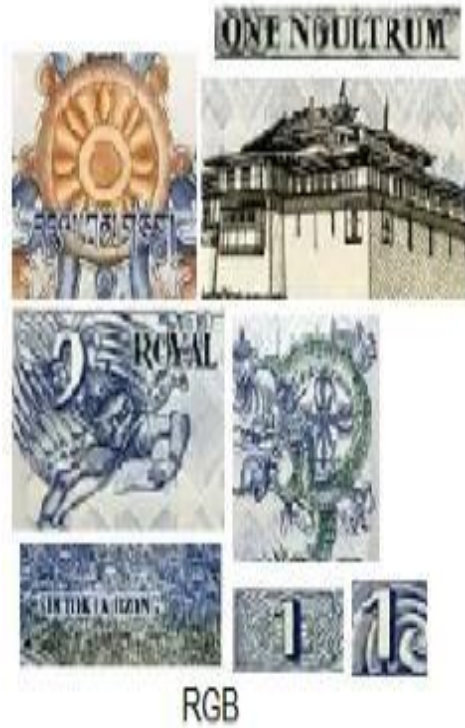

RGB

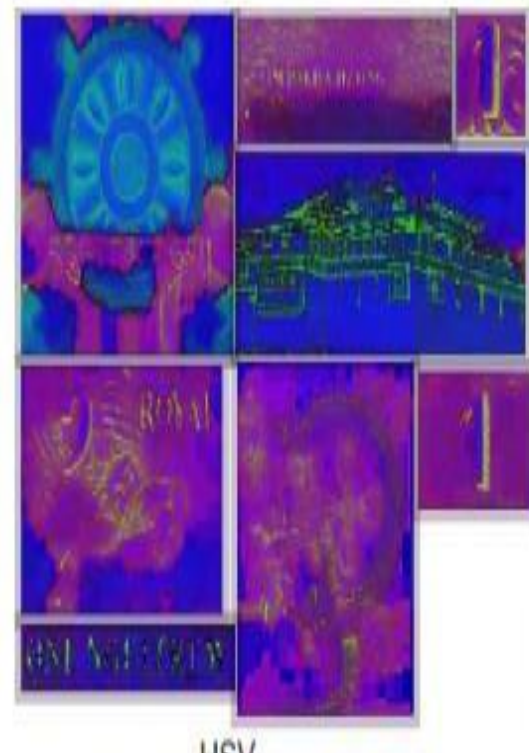

HSV
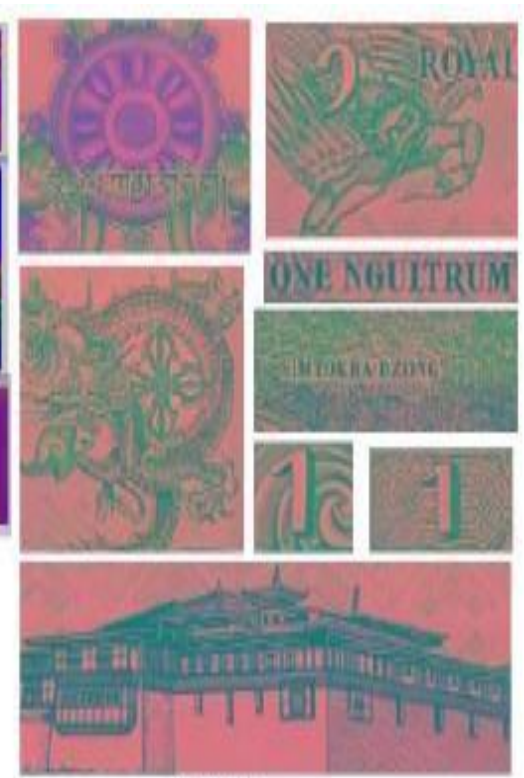

$\mathrm{YCbCr}$

Fig 6: Different feature Segments Bhutan Currency

As shown in above Figure 6 eight segments features of each type of color image have been considered for detecting the Bhutan currency. 


\begin{tabular}{|c|c|c|c|c|c|}
\hline MR1 & MG1 & MB1 & MM1 & SD1 & Corr1 \\
\hline 138.30102 & 148.0043 & 152.97226 & 146.42586 & 51.445079 & 0.9948078 \\
\hline 155.33293 & 162.79661 & 168.4468 & 162.19211 & 61.385866 & 0.99606 \\
\hline 113.67898 & 125.84739 & 136.06492 & 125.1971 & 48.373529 & 0.9907734 \\
\hline 142.59035 & 146.1875 & 136.48268 & 141.75351 & 72.589403 & 0.9971904 \\
\hline 160.32399 & 157.39998 & 142.43283 & 153.3856 & 78.703277 & 0.9979759 \\
\hline 131.03292 & 139.61853 & 150.85536 & 140.50227 & 68.493061 & 0.9974056 \\
\hline 182.99961 & 161.65765 & 143.02073 & 162.55933 & 60.517242 & 0.9955494 \\
\hline 156.25529 & 166.24388 & 171.17458 & 164.55791 & 60.246289 & 0.9953973 \\
\hline 1180.5151 & 1207.7558 & 1201.4502 & 1196.5737 & 501.75375 & 7.9651598 \\
\hline MR2 & MG2 & MB2 & MM2 & SD2 & Corr2 \\
\hline 0.5421139 & 0.1279367 & 0.6022634 & 0.4241047 & 0.254194 & 0.9893667 \\
\hline 0.4889168 & 0.1386797 & 0.6691012 & 0.4322992 & 0.2859596 & 0.9866141 \\
\hline 0.4989198 & 0.2118067 & 0.5454226 & 0.4187164 & 0.2170473 & 0.9891 \\
\hline 0.2275798 & 0.1279058 & 0.5734368 & 0.3096408 & 0.2808309 & 0.9661385 \\
\hline 0.1471735 & 0.1925787 & 0.6300651 & 0.3232724 & 0.3089862 & 0.9754655 \\
\hline 0.5485171 & 0.2171556 & 0.5965011 & 0.4540579 & 0.2661683 & 0.989898 \\
\hline 0.2306094 & 0.2834234 & 0.738168 & 0.4174003 & 0.3164511 & 0.9882401 \\
\hline 0.4987469 & 0.1315092 & 0.6791181 & 0.436458 & 0.2843304 & 0.9797053 \\
\hline 3.1825771 & 1.4311956 & 5.0340763 & 3.2159497 & 2.2139678 & 7.8645282 \\
\hline MR3 & MG3 & MB3 & MM3 & SD3 & Corr3 \\
\hline 141.10698 & 131.67586 & 123.44531 & 132.07605 & 26.802261 & 0.9717149 \\
\hline 154.44782 & 131.55934 & 124.36677 & 136.79131 & 33.588241 & 0.9781196 \\
\hline 121.95696 & 134.33049 & 121.86156 & 126.04967 & 24.341085 & 0.958139 \\
\hline 139.67697 & 124.37823 & 127.03091 & 130.36204 & 36.767592 & 0.9844172 \\
\hline 150.46436 & 121.07533 & 130.3213 & 133.95366 & 40.871235 & 0.9892883 \\
\hline 134.80421 & 134.13257 & 123.45874 & 130.79851 & 34.980099 & 0.9854062 \\
\hline 158.49375 & 116.63359 & 138.68219 & 137.93651 & 34.099488 & 0.9816732 \\
\hline 156.69133 & 131.62111 & 123.30069 & 137.20438 & 33.218867 & 0.9769823 \\
\hline 1157.6424 & 1025.4065 & 1012.4675 & 1065.1721 & 264.66887 & 7.8257407 \\
\hline & & & & & \\
\hline
\end{tabular}

Fig 7: Data set of original currency of Bhutan

\begin{tabular}{|c|c|c|c|c|c|}
\hline MR1 & MG1 & MB1 & MM1 & SD1 & Corr1 \\
\hline 138.71221 & 142.51818 & 147.47465 & 142.90168 & 58.543773 & 0.0343695 \\
\hline 179.50677 & 176.23999 & 176.38125 & 177.376 & 66.234789 & 0.2896358 \\
\hline 121.65754 & 126.14439 & 137.12067 & 128.30753 & 62.486935 & 0.2199479 \\
\hline 149.96739 & 146.92442 & 141.98471 & 146.29218 & 80.760568 & 0.0228248 \\
\hline 177.67141 & 162.15155 & 152.64118 & 164.15471 & 81.17572 & 0.7152342 \\
\hline 141.13828 & 141.98134 & 144.17164 & 142.43042 & 72.034172 & -0.064191 \\
\hline 206.48338 & 172.64952 & 152.33647 & 177.15646 & 69.318202 & 0.4813872 \\
\hline 176.66872 & 175.25824 & 175.0258 & 175.65092 & 70.415167 & 0.2653052 \\
\hline 1291.8057 & 1243.8676 & 1227.1364 & 1254.2699 & 560.96933 & 1.9645141 \\
\hline MR2 & MG2 & MB2 & MM2 & SD2 & Corr2 \\
\hline 0.5590586 & 0.098286 & 0.5789791 & 0.4121079 & 0.2831517 & 0.1684083 \\
\hline 0.321928 & 0.0842817 & 0.719597 & 0.3752689 & 0.3578204 & 0.4941908 \\
\hline 0.4633799 & 0.1894235 & 0.5474295 & 0.4000776 & 0.2729258 & 0.7566413 \\
\hline 0.1000563 & 0.1407784 & 0.588579 & 0.2764712 & 0.3211468 & -0.01122 \\
\hline 0.0634284 & 0.2358675 & 0.6967513 & 0.3320158 & 0.3505873 & 0.2884802 \\
\hline 0.4095196 & 0.1346167 & 0.5747611 & 0.3729658 & 0.3206041 & 0.1640008 \\
\hline 0.2186742 & 0.2964542 & 0.8132984 & 0.4428089 & 0.3767156 & 0.6378955 \\
\hline 0.3609551 & 0.0788355 & 0.7039859 & 0.3812588 & 0.3591812 & 0.4298405 \\
\hline 2.4970002 & 1.2585434 & 5.2233814 & 2.992975 & 2.6421329 & 2.9282377 \\
\hline MR3 & MG3 & MB3 & MM3 & SD3 & Corr3 \\
\hline 137.90242 & 130.69436 & 126.05022 & 131.549 & 29.507992 & 0.0204631 \\
\hline 168.21693 & 127.67439 & 129.39537 & 141.76223 & 38.171007 & 0.1814327 \\
\hline 124.26182 & 133.45469 & 125.22239 & 127.6463 & 31.104549 & 0.2752669 \\
\hline 142.48505 & 125.33356 & 129.71162 & 132.51008 & 40.791793 & 0.0240451 \\
\hline 158.31395 & 121.53885 & 135.44464 & 138.43248 & 42.886907 & 0.7192594 \\
\hline 137.93942 & 129.07133 & 127.48266 & 131.49781 & 36.184483 & -0.061203 \\
\hline 170.97741 & 114.08405 & 144.32195 & 143.1278 & 40.61997 & 0.5300772 \\
\hline 166.85809 & 127.70632 & 128.66042 & 141.07494 & 39.596374 & 0.2276081 \\
\hline 1206.9551 & 1009.5575 & 1046.2893 & 1087.6006 & 298.86307 & 1.9169496 \\
\hline & & & & & \\
\hline
\end{tabular}

Fig 8: Data set of Fake currency of Bhutan

Here, mean of each values are shown in Figure 7 and 8 MR1,MG1,MB1,MM1 SD1 and Corr1 (Mean Red, Mean Green, Mean Blue, Mean of Mean RGB, Standard Deviation and Correlation of RGB Image) have calculated for the processing of image on Bhutan currency. Same way calculated this for $\mathrm{YCbCr}$ and HSV image also.

\section{CONCLUSION}

In this Research, on bank note identification and detection technique using deep learning is considered. Also focused Bank note of various countries by extracting its features in depth and analyze it using deep learning based algorithm and also recommend that this technique can be used to detect forged bank note by persons to avoid personal monetary damages.

Proposed method is tested by giving duplicate as well as original note as input. through this results are tested for both the cases i.e. front view and back view and it properly works. Proposed system used MATLAB tool on the basis deep learning process of automatic recognition of fake and genuine Indian and Foreign currencies. Proposed system is cost effective and efficient system than the existing one.

\section{REFERENCES}

[1] Achal Kamble , Prof. M. S. Nimbarte, "Design and Implementation of Fake Currency Detection System", International Journal on Future Revolution in Computer Science \& Communication Engineering, Volume 4, Issue 4,pp. 400- 405, 2018.

[2] Tushar Agasti et al., "Fake currency detection using image processing", IOP Conf. Series: Materials Science and Engineering, 2017.

[3] Monali Patil et al., "Fake Currency Detection using Image Processing", International Journal on Future Revolution in Computer Science \& Communication Engineering, ISSN: 2454-4248, Volume: 4 Issue: 4, pp. $865-868,2018$

[4] Gai, S., et.al.,'Employing quaternion wavelet transform for banknote classification." Neurocomputing ,2013, 118:171-178

[5] Bhurke, C.; Sirdeshmukh, M.; Kanitkar, M.S. Currency recognition using image processing. Int. J. Innov. Res. Comput. Commun. Eng. ,pp. 4418-4422, 2015.

[6] Chae, et. al , "The Study for Authenticity Distinguish of Bank note using UV Information," Proceedings of KIIT Summer Conference, pp. 753-756, 2009.

[7] Lee, G. H., and Park, T. H., 2011, "Automatic Extraction of UV patterns for Paper Money Inspection," Journal of Korean Institute of Intelligent Systems, 21 (3), pp. 365371.

[8] Syed ejaz ali, " challenges in Indian currency denomination recognition \& authentication", international journal of research in engineering and technology, 2014.

[9] Tuyen Danh Pham, et al., "Deep Learning-Based Multinational Banknote Type and Fitness Classification with the Combined Images by Visible-Light Reflection and Infrared-Light Transmission Image Sensors”, 2018.

[10] Krizhevsky, et al., "ImageNet classification with deep convolutional neural networks", Communication of the ACM. Pp.84-90. 2017.

[11] Dr. Yogesh Kumar Sharma, "security-in-digital-imagesusing-visualcryptography-scheme",Journal of Computational Information Systems, ISSN: 1553-9105, Volume No. 14, Issue No. 6, pp. 49-57, Nov. 2018.

[12] Dr. Yogesh Kumar Sharma, "An Empirical Research Framework for Encryption Authentication with Image and Video" ,Journal of Advance Research in Dynamical and Control Systems, ISSN: 1943- 023X, Volume No. 10, Issue No. 8, pp. 59-71, 2018. 